\title{
TECNICA DE GAMMA SCANNING PARA EL DIAGNÓSTICO EN OPERACIÓN, DE EQUIPOS DE REFINACIÓN DE PETRÓLEO Y GAS NATURAL
}

\author{
GAMMA SCANNING TECHNIQUE FOR DIAGNOSTING OIL \\ AND NATURAL GAS REFINING EQUIPMENT IN OPERATION
}

Carlos Sebastián Calvo ${ }^{1}$

RECEPCIÓN: 03 DE SETIEMBRE DE 2020

ACEPTACIÓN: 27 DE SETIEMBRE DE 2020

\section{RESUMEN}

Las técnicas de radioisótopos son muy útiles para investigar la variedad de problemas en la industria de refinación de petróleo, gas natural y petroquímica. Estas técnicas y sus aplicaciones están al servicio rutinario de la industria en muchos países. La inspección con rayos gamma, referida a menudo como «perfilaje de columna», es una técnica conveniente, rentable, rápida, eficaz y no invasiva, para examinar detalles interiores de una columna de proceso o de un recipiente de reacción química, mientras está en funcionamiento. El perfilaje de una columna permite realizar instalaciones de procesamiento de gas y plantas químicas, el estudio de la hidráulica en bandejas o platos o empaques dentro de una columna de destilación en cualquier de condición fijada en línea. Asimismo, proporciona datos esenciales para optimizar el rendimiento de las columnas, prolongar su tiempo de funcionamiento, manejar los efectos de deterioro de desempeño, de deposición de sólidos y suciedad e identificar las necesidades de mantenimiento antes o luego de las paradas programadas. Esta información en línea puede reducir significativamente tiempos de reparación. Por otro lado, es posible determinar el estado operativo de un contenedor que aloje en su interior un material hidrogenado, inspeccionando desde el exterior el estado interno, mediante el perfilaje neutrónico. La condición fundamental para su aplicación es que haya material hidrogenado en contacto con la otra cara de la pared. También es posible medir niveles desde el exterior de un recinto con materiales hidrogenados o de número atómico bajo como niveles de ácidos u otros materiales corrosivos o peligrosos. Otra alternativa es la detección de interfases entre fluidos dentro de estanques.

Palabras clave: Columnas industriales, escaneo, radioisótopo, fuente, detector, empaque, neutrón, retrodispersión.

\section{ABSTRACT}

Radioisotope techniques are highly useful tools to investigate variety of problems in oil \& natural gas refining and petrochemical industry. These techniques and applications are in routine service to industries of many countries. Gamma-ray scanning, often referred to as "column scanning", is a convenient, cost effective, fast, efficient and non-intrusive technique to examine inner details of a process column or a reaction vessel, while it is in operation. Column scanning allows an engineer in petroleum refineries, gas processing installations and chemical plants, to study tray or packing hydraulics inside a distillation column at any set of on-line condition. It provides essential data to optimize the performance of columns, extend column run times, track the performancedeteriorating effects of fouling and solids deposition, and identify maintenance requirements well in advance of scheduled turnarounds. This on-line information can reduce repair downtimes significantly. On the other hand, it is possible to determine the operational state of a container hosting inside a hydrogenated material, inspecting the internal condition, using the neutron logging from the outside. Neutron backscatter can be the solution in these cases. Either way, the fundamental condition for its application is the existence of hydrogenated material in contact with the other side of the wall. It is also possible to measure levels from the outside of an enclosure with hydrogenated materials or low atomic number; for example, levels of acids or other corrosive or hazardous materials. Another alternative is the detection of interfaces between fluids inside tanks. Column scanning were introduced a few years ago. Since then, the gamma scanning technique is largely used in routine services. Qualified technical staffs have performed hundreds of scans on various types of process columns in petroleum refineries, gas processing installations and chemical plants. Keywords: Industrial columns, scanning, radioisotope, source, detector, pack, neutrón, backscatering.

1 Universidad Ricardo Palma, carlos.sebastian@urp.edu.pe 


\section{INTRODUCCIÓN}

Los radioisótopos fueron aplicados inicialmente para resolver problemas en la industria desde mediados del siglo pasado. Básicamente, se han empleado en la optimización de los procesos en las plantas industriales. Los beneficios económicos que se derivan de la tecnología de los radioisótopos son considerables; sin embargo, no ha sido reconocida por los gobiernos de países en desarrollo. Cuantificando el desarrollo de los Estados miembros de la Agencia Internacional de Energía Atómica (IAEA, por sus siglas en inglés), cerca de cincuenta de ellos tienen grupos en el área de aplicaciones radioisotópicas. El presente reporte tiene como objetivo presentar lo más reciente en técnicas de gamma scanning y retrodispersión neutrónica en la inspección de problemas en columnas, tanques, y en muchos procesos del sector industrial. De esta manera, no solo se proporcionará una descripción de lo que puede ser llevado a cabo mediante la aplicación de fuentes selladas de radioisótopos, sino también se divulgarán todos los aspectos de diseño.

Las industrias de procesos químicos y petroquímicos son los principales usuarios y beneficiarios de la tecnología de radioisótopos. Estas técnicas son muy competitivas y ampliamente aplicadas en la detección de fallas y en los procesos de análisis técnicamente complejos en plantas industriales que operan continuamente. El éxito de estas aplicaciones es atribuido a su capacidad única de proporcionar información que, de otra manera, no podría ser obtenida por técnicas alternativas.

Las mejores técnicas de fuentes radioisotópicas selladas y sus aplicaciones aquí presentadas son gamma scanning de columnas, y niveles y detección de interfases usando técnica de retrodispersión de neutrones. Estas técnicas ampliamente usadas ayudan a obtener una clara "visión interior" de los equipos de tratamiento en los procesos de detección de fallas, sin el costo excesivo de una parada. Son más útiles en las técnicas en línea y no invasivas para detectar fallas y optimizar el desempeño de la unidad.

Los ejemplos descritos en este artículo claramente demuestran el valor potencial de las aplicaciones de los radioisótopos en la industria. Probablemente, una relación costo-beneficio promedio de 20:1-50:1 es razonablemente representativo. Hay pocas inversiones a corto plazo que proporcionarán un retorno de esta magnitud. El costo efectivo de las aplicaciones de los radioisótopos debería ser ampliamente divulgada para motivar a los industriales a que tomen total ventaja de esta tecnología. Lamentablemente, la industria no utiliza esta tecnología más extensamente de lo que se ha hecho hasta el presente.

\section{FUNDAMENTO TEÓRICO}

$\mathrm{Al}$ interponerse un cuerpo entre un emisor y un detector de radiación gamma, la intensidad de radiación original es atenuada puesto que los átomos del material interpuesto y los fotones gamma interactúan. A equivalencia de otras variables, la mitigación de la radiación sufrida resulta ser función de la densidad del cuerpo obstaculizante.

Los perfilajes gamma a efectuar consisten en trasladar, simultáneamente, una fuente de radiación y un detector a lo largo de la columna bajo estudio. Ambos se sitúan (aunque no es la única opción) opuestas diametralmente, y queda entre ambos el material interpuesto. Cualquier componente que se encuentre internamente debilitará el haz de radiaciones en diferente grado, por lo que se mostrará el resultado del detector versus la altura a fin de conseguir el perfil de densidad vertical.

La presencia de barreras que afectan la normal operación de una torre derivará en diversas variaciones en el perfil de densidad. Las causas de mal funcionamiento de una torre pueden ser identificadas a través de un análisis de perfil gamma. Se analizarán diversas anomalías probables. Podría, por ejemplo, considerarse el desplome de un plato (desprendimiento total o parcialmente), inundación (un obstáculo frena u obstaculiza el tránsito del líquido hacia el inferior), arrastre (la presión entre platos 
consecutivos es alta, de tal manera que la parte líquida que entra es arrastrada hacia arriba combinando los líquidos con gases), lluvia (movimiento de líquido a través de los conductos de gas, desde la zona activa hacia debajo de un plato), formación de espuma (se forma ante la aparición de burbujas de gas en la superficie del líquido y dificulta el desplazamiento del gas hacia arriba, lo que reduce la calidad de separación del plato).

El dispositivo básico para perfilaje gamma consiste en un sistema de movimiento semi automático que permite mover la fuente radiactiva y el detector de radiaciones simultánea y longitudinalmente a lo largo de la columna, ubicados en forma opuesta diametralmente. El conjunto se completa con un sistema de captura de datos adaptado a un equipo computador. Las actividades de trabajo de campo empiezan con la implementación de un "sistema de movimiento", que está conformado por un arreglo metálico de carretes, que son controlados por manijas que permiten enrollar los cables de acero donde se fijarán la sonda detectora y el porta fuentes. En la parte alta de la estructura se fijan dos sujetadores por donde se pasan los indicados cables, quedando el detector y la fuente en la parte baja de la torre.

Seguidamente se fija el porta fuentes en uno de los extremos del cable de acero, considerando que el otro ya se encuentra fijado a la sonda detectora. Posteriormente se realiza la medición en un área cercana a la base de la columna. Finalmente se procede a obtener el perfil utilizando la fuente y la sonda y se accionan sus respectivas manijas. Se deben realizar los movimientos en intervalos fijos, recomendándose usar $5 \mathrm{~cm}$ o $10 \mathrm{~cm}$, dependiendo de las dimensiones de las columnas.

Una vez concluido el barrido, se procede a desmontar el sistema. La información recolectada permanecerá almacenada en el computador para su posterior análisis.

La figura 1 nos muestra un típico perfil, con probables anormalidades que se puedan presentar.

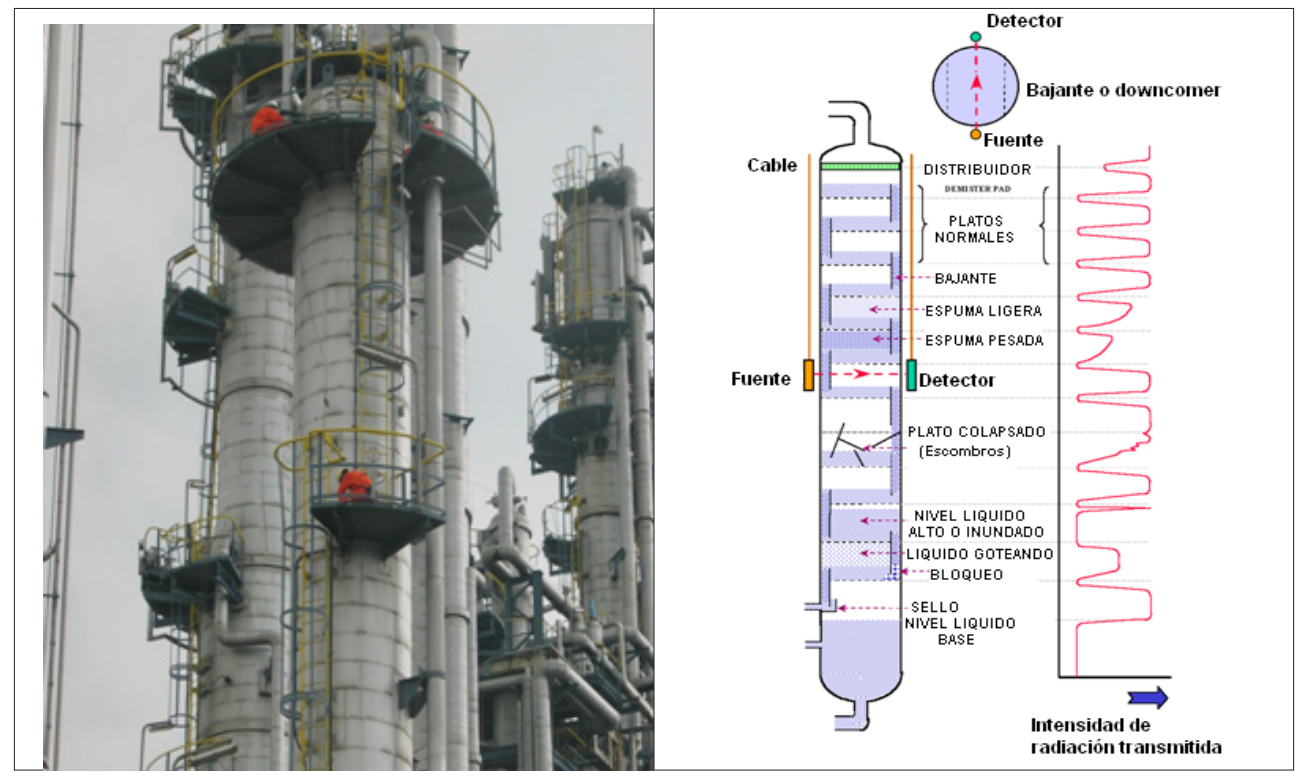

Fig. 1 Aplicación de la técnica de gamma scanning y perfil de densidades que muestran las posibles señales de anomalías que se pueden encontrar en una columna de fraccionamiento.

\subsection{Gamma scanning de columnas}

El gamma scanning es la mejor técnica para realizar una inspección interna de algunos equipos en los procesos, sin interrupción de la producción. Un haz colimado de rayos gamma penetrantes se deja 
pasar través de las paredes de un recipiente modificado en su interior, y sale por el lado opuesto. A partir de la medición de la intensidad de la radiación transmitida, información valiosa se puede obtener acerca de las densidades de los materiales presentes dentro del recipiente. A mayor densidad del material, menor es la radiación que pasa a través del mismo, así que los rayos gamma más significativos (intensos) son transmitidos a través del vapor comparado con la fase líquida.

El scanning de densidad de columnas de destilación, es la aplicación más frecuentemente usada de esta técnica. Sin afectar la unidad de procesamiento esta técnica confiable y precisa puede ser empleada para determinar:

- El nivel de líquido en platos.

- La presencia o ausencia de estructuras internas, tales como platos, absorbentes (filtros), empaques y distribuidores.

- La extensión y posición de inyección y acumulación de líquido por desbordamiento.

- La posición y la densidad característica de espumas.

- Presencia y formación de coque.

- Localización y extensión de inundaciones.

- Bloqueos causados por obstrucción de los bajantes, suciedad en los platos, lodo o carga alta de líquido en los platos.

- Nivel de líquido en los platos.

La fuente de rayos gamma y los detectores de radiación son movidos hacia abajo simultáneamente en los lados opuestos de la columna. La intensidad es almacenada a intervalos apropiados y el perfil del estado de operación instantáneo grafica la respuesta del detector versus la altura de la columna. La estructura del plato y del líquido en el plato producen alta absorción, mientras que la presencia de la espuma y el arrastre moderan ligeramente el perfil del vapor esperado. Estudios sobre el grado de formación de espuma, pueden ser realizados para generar perfiles de densidad a diferentes concentraciones de antiespumante agregado.

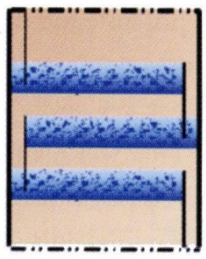

Pasa Bandeja Simple

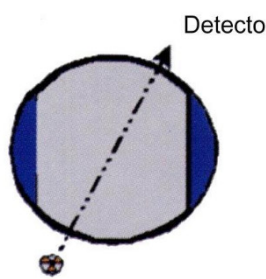

Fuente Radioactiva

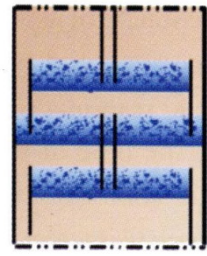

Pasa Bandeja Doble Dos Pasa Bandejas

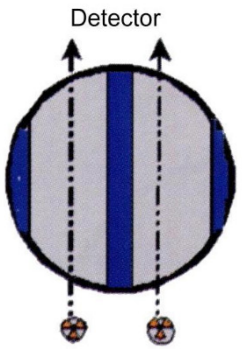

Fuente Radioactiva

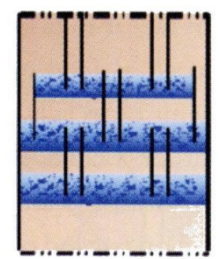

Pasa Bandeja Cuadruple Multi Pasa Bandeja

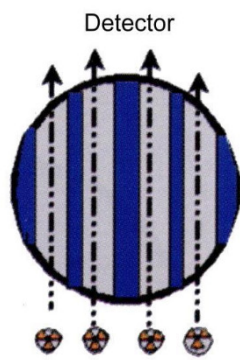

Fuente Radioactiva

Figura 2. Orientaciones típicas fuente-detector a usar y vista de las lineas de radiación transmitida. 
Los siguientes procedimientos se realizarán para montar el gamma scanning:

- Obtención detallada de los esquemas mecánicos de la columna.

- Solicitud de asistencia en el proceso o del ingeniero químico para los detalles del proceso.

- Establecimiento de la línea de orientación del scan y el número de scanning.

- Chequeo de la fuente y del detector para que la alineación sea cada $50 \mathrm{~cm}$ del scanning.

- Verificar alguna disminución inusual en la intensidad de la radiación debido a obstrucción externa en la columna.

Una típica línea recomendada de orientación del scan para las columnas con platos como las que son mostradas en la figura 2.

\subsubsection{Aspectos metodológicos del scanning de rayos gamma}

El diseño experimental de un gamma scanning se puede observar en la siguiente figura:

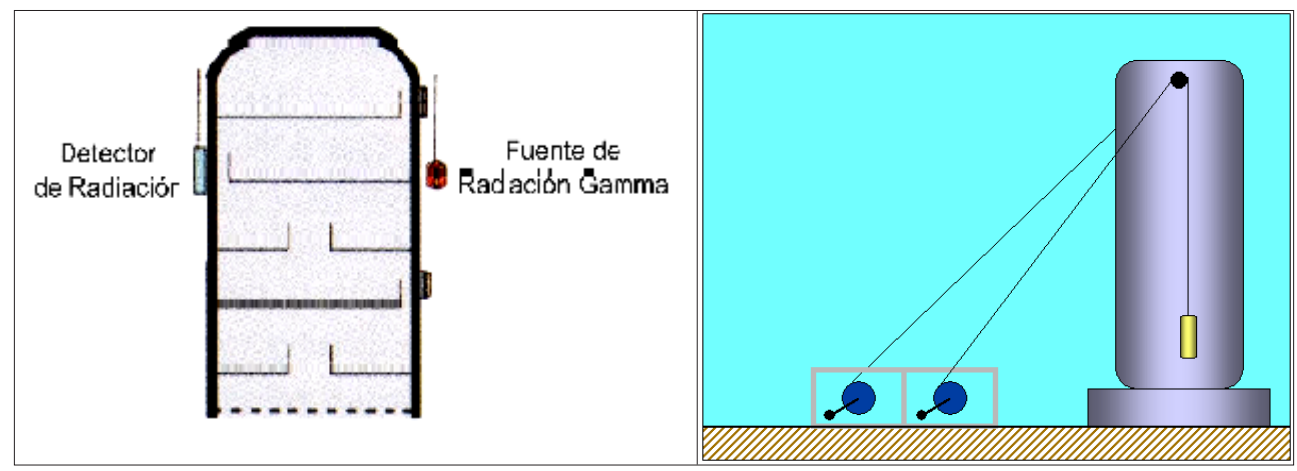

Figura 3. Configuración del sistema fuente-detector en un scanning gamma de una columna. Elaboración propia.

La fuente radioactiva es colocada dentro de un colimador panorámico $\left(360^{\circ}\right)$ figura 4.

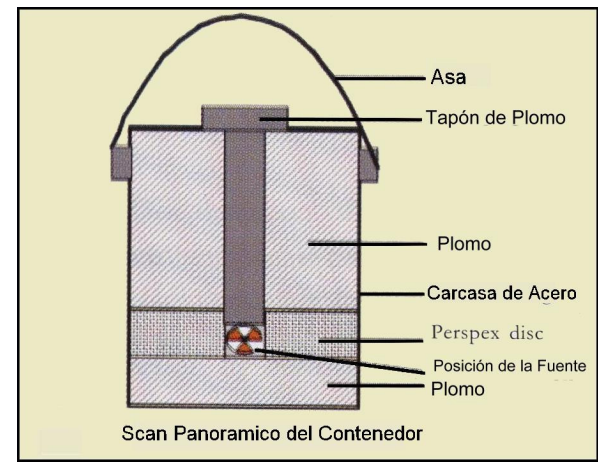

Figura 4. Colimador panorámico de fuente.

Los resultados del scanning pueden ser discutidos inmediatamente con los ingenieros de procesos de manera que se pueda realizar las correcciones necesarias. 
INDUSTRIAL

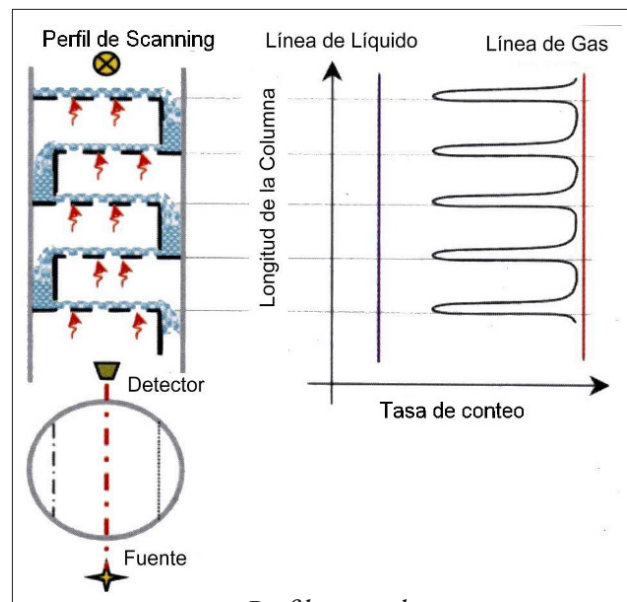

Perfil normal

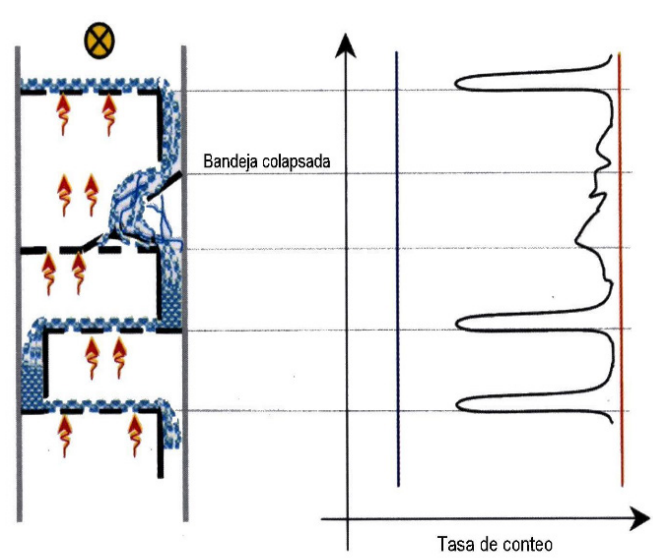

Perfil con plato caído

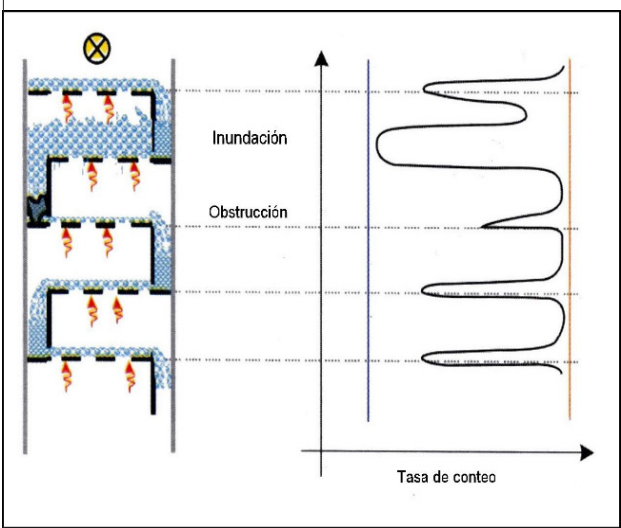

Perfil con inundación

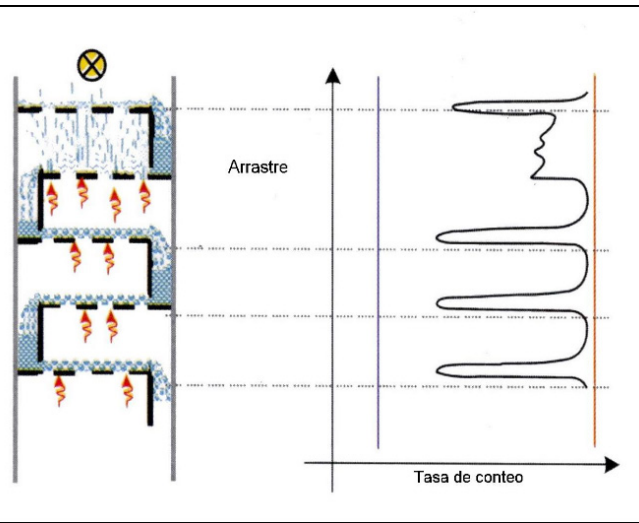

Perfil con arrastre

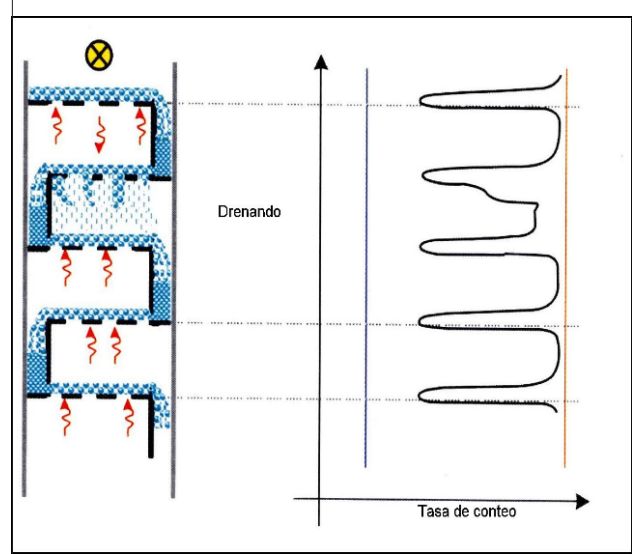

Perfil con drenaje (lluvia)

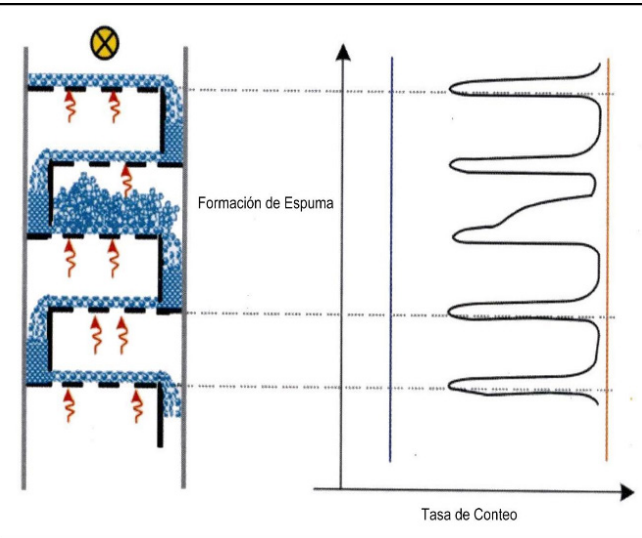

Perfil con espuma

Figura 5. Perfiles típicos en el gamma scanning. 
La siguiente información previa es útil:

- Perfiles del gamma scanning de una columna "vacía" (con toda su estructura interna, pero sin estar operando).

- Perfil del scanning antes de una parada de mantenimiento.

- Perfil del scanning después de una parada de mantenimiento cuando la columna está en condición de operaciones normales.

Hay clara diferencia entre un scan limpio (seco) y uno normal (húmedo), como se ve en la figura a continuación.

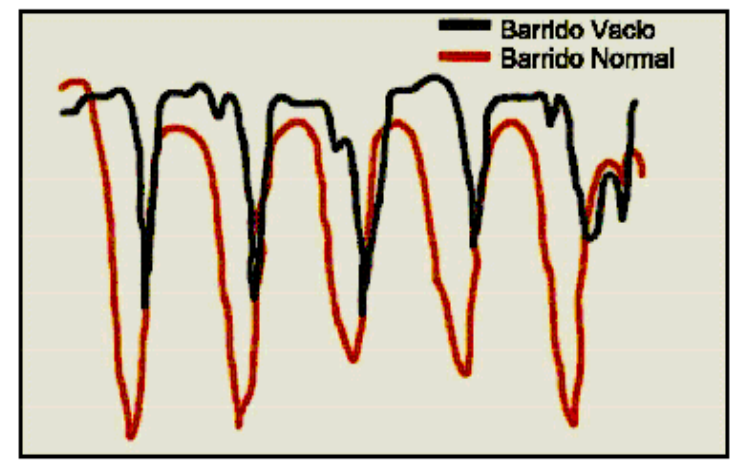

Figura 6. Ejemplo de perfilajes de barrido de vacio (seco) y normal (húmedo).

Esto es muy importante para las comparaciones que sean llevadas a cabo a lo largo de las mismas líneas de scanning (líneas entre la fuente radioactiva y el detector). Las medidas pueden ser tomadas a intervalos pequeños (menor o igual a $50 \mathrm{~mm}$ ).

\subsection{Scanning por coordenadas}

Algunas columnas para perfilar en esta oportunidad cuentan con lecho fijo o mixto. El scanning por coordenadas será el recomendable para las columnas de lecho fijo o relleno. Una orientación típica de líneas coordenadas del scan es mostrada en la figura 7. Al menos dos scannings serán necesarios para analizar las columnas de lecho fijo. Columnas de diámetros grandes se perfilarán de manera diferente, por lo que son necesarios al menos cuatro barridos.

El scanning por coordenadas puede ser utilizado para investigar condiciones relacionadas con los procesos tales como:

- Obstrucciones o desbordamientos.

- Entrada o transporte de líquido.

- Distribución desigual de fluido a través de los lechos fijos.

El scanning por coordenadas también puede ser usado para investigar problemas mecánicos de construcción tales como:

- Colapso de lechos fijos o

- Distribución no uniforme de material de empaque. 


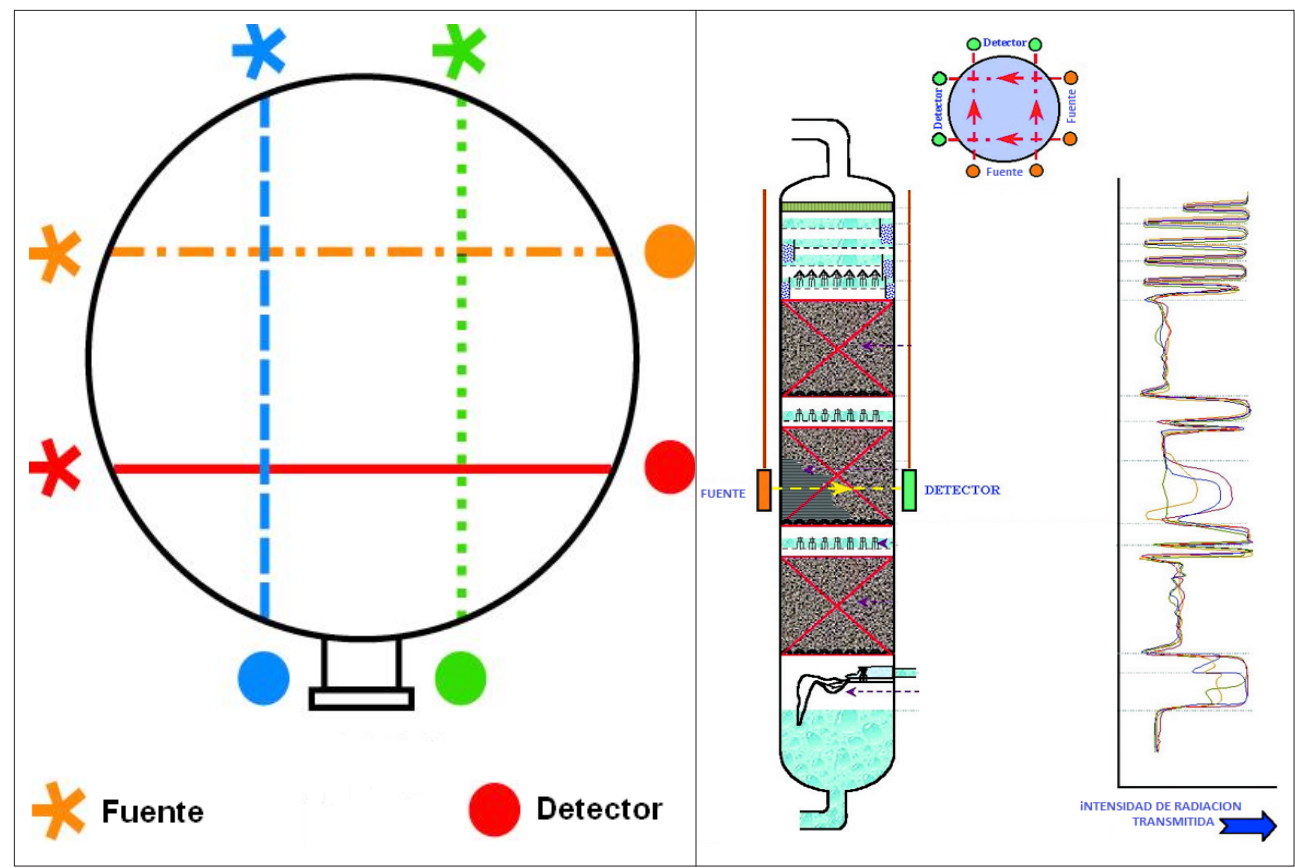

Figura 7. Orientaciones de la cuadricula de las líneas de barrido, aplicable a columnas de lecho empacado en el scanning por coordenadas. Elaboración propia.

El scanning por coordenadas será útil también para investigar la instalación correcta de distribuidores, así como también la correcta distribución del líquido de alimentación que entra. Un distribuidor irregular puede minar el desempeño de todo el lecho empacado y la columna. Los distribuidores de líquido, deben distribuir el fluido uniformemente sobre el lecho, resistir obstrucciones y sucio, y también proporcionar espacio libre para el flujo de gas. Un incorrecto nivel de agua en el distribuidor instalado, el cual es inclinado, puede ocasionar que el flujo del líquido sea preferentemente en un solo lado de la columna.

\subsection{Retrodispersión de neutrones}

Neutrones de alta energía o "rápidos" de una fuente radioactiva son dirigidos hacia un contenedor.

Neutrones rápidos se ponen lentos principalmente por colisiones con átomos de hidrógeno del material dentro del contenedor. Como la fuente y el detector se mueven hacia abajo a ambos lados del recipiente, las interfases pueden ser detectadas dado que ellas involucran un cambio en la concentración de átomos de hidrógeno en el contenido del recipiente. Una parte de los neutrones térmicos se regresan hacia la fuente. Con un detector térmico de neutrones cerca de la fuente, estos neutrones por retrodispersión pueden ser medidos. El número de neutrones por retrodispersión es directamente proporcional a la concentración de átomos de hidrógeno que están frente al detector de neutrones. Como la fuente y el detector se mueven hacia abajo al lado del contenedor, las interfases pueden ser detectadas siempre que ellos involucren un cambio en la concentración de átomos de hidrógeno.

Las mediciones de la retrodispersión de neutrones claramente indica enlaces sólido/líquido y líquido/líquido y, con interpretación cuidadosa de los datos, los niveles de espuma. La inspección de 
la interfase entre el crudo y el agua, así como también la cantidad de las fracciones de hidrocarburo es la mejor aplicación de esta técnica. Con tal de que el contenedor tenga un espesor de pared menor de $100 \mathrm{~mm}$, el uso de neutrones es una técnica rápida y versátil, y es ideal si el acceso a ambos lados del contenedor no es posible.

Las aplicaciones de la técnica de retrodispersión de neutrones incluyen:

- Inventario de tanques de almacenamiento de crudos sin aforo

- Calibración sin contacto o medidas convencionales de niveles

- Determinación de capas de lodo y agua en los tanques

- Medición de niveles de empacado en las torres de absorción

- Identificar obstrucciones o acumulaciones en tuberías y reactores

- Medida de los niveles de catalizador en reactores

- Localizar interfases de agua /compuestos orgánicos

- Medir niveles de espuma

- Determinar niveles absorción en torres empacadas

- Calibrar indicadores de nivel rápida y fácilmente

Los detectores de neutrones de helio (He-3) o BF3 pueden ser usados. El detector de $\mathrm{He}-3$ tiene una alta eficiencia y es el más utilizado en la medición de neutrones actualmente.

La fuente de neutrones principalmente utilizada es ${ }^{241} \mathrm{Am} / \mathrm{Be}$ de $1 \mathrm{Ci}$, la cual produce un flujo de $2,2 \times 10^{6} \mathrm{n} / \mathrm{s}$ con energías desde $0,1 \mathrm{MeV}$ a $11,2 \mathrm{MeV}$, y promedio de energía de aproximadamente 5 $\mathrm{MeV}$. La fuente de neutrones Cf-252 es también utilizada, pero es más costosa.

TABLa 1: Fuentes selladas de neutrones comúnmente usadas

\begin{tabular}{|c|c|c|c|}
\hline Fuente de neutrones & $\mathrm{T}_{1 / 2}$ & $\begin{array}{c}\text { Energía promedio del } \\
\text { neutrón }(\mathrm{MeV})\end{array}$ & $\begin{array}{c}\text { Flujo o rapidez de los } \\
\text { neutrones }\end{array}$ \\
\hline $\mathrm{Am} 241-\mathrm{Be}$ & $433 \mathrm{a}$ & 4,46 & $2,6 \times 10^{6} \mathrm{n} / \mathrm{C} \mathrm{i} / \mathrm{s}$ \\
\hline $\mathrm{Cf}-252$ & $2,65 \mathrm{a}$ & 2,12 & $2,3 \times 10^{6} \mathrm{n} / \mathrm{mg} / \mathrm{s}$ \\
\hline
\end{tabular}

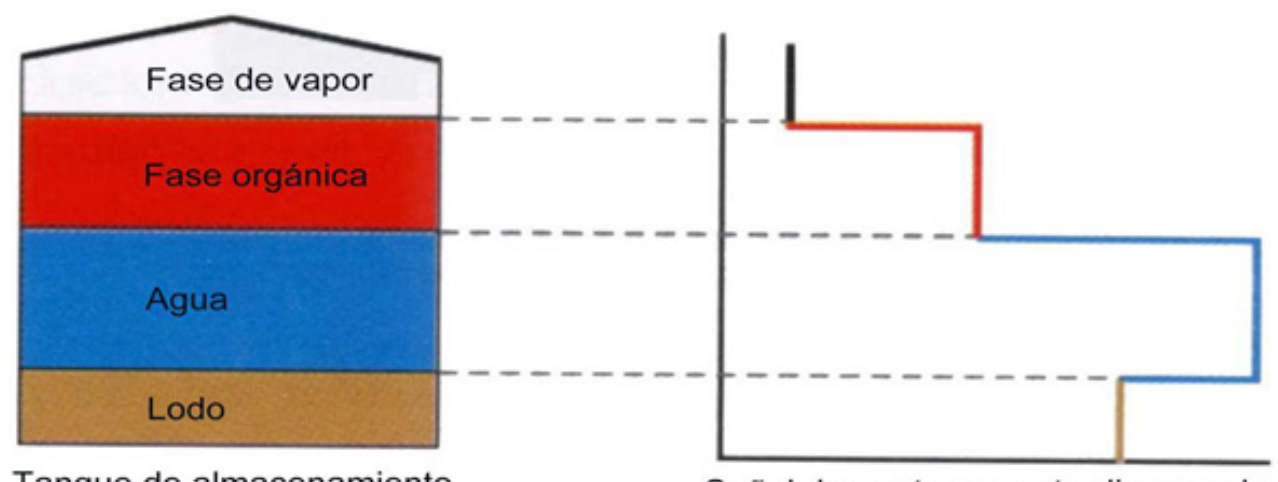

Tanque de almacenamiento

Señal de neutrones retrodispersados

Figura 8. Respuesta del detector en la retrodispersión de neutrones.

La retrodispersión de neutrones también puede ser usada para medir el nivel y la interfase de líquidos transportados en tuberías. 


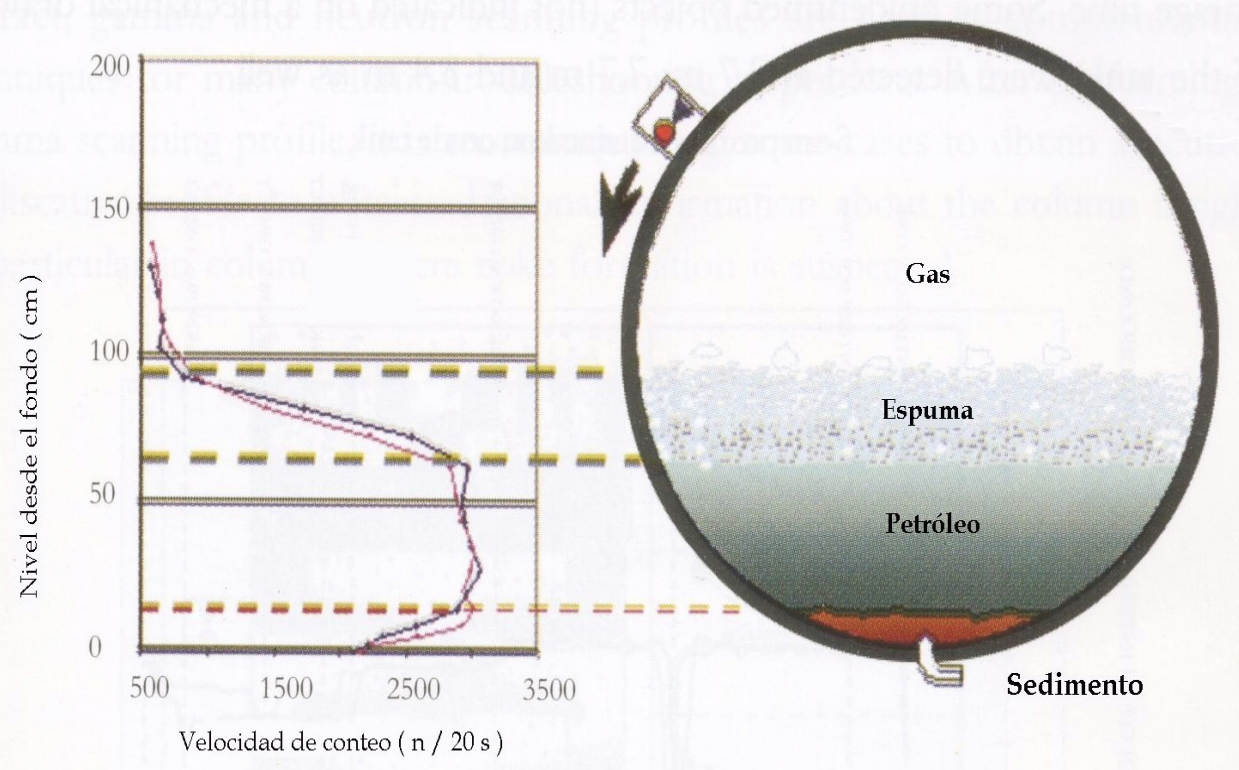

Figura 9. Perfil de la retrodispersión de neutrones dentro de una tubería de petróleo.

\section{CONCLUSIONES}

Los ejemplos descritos demuestran claramente el valor potencial de la aplicación de los radioisótopos a escala industrial.

El gamma scanning es la única herramienta de diagnóstico disponible que puede ser aplicada con confianza en cualquier proceso de destilación para obtener el comportamiento hidráulico real del sistema.

El desarrollo de las soluciones planteadas a los problemas de operación industrial mencionados, es posible, porque se basa en la capacidad que tiene la tecnología de los radioisótopos de proporcionar beneficios económicos sustanciales con la pequeña inversión que se hace.

Los argumentos económicos son solo la forma cuantitativa por la cual los expertos en radioisótopos pueden persuadir a los industriales a invertir en la tecnología.

El estudio de los beneficios económicos es importante y trabajadores en este campo podrían esforzarse para evaluarlos y publicar casos exitosos de estudio.

\section{REFERENCIAS}

[1] J. Abdullah, "Gamma Scanning for Troubleshooting, Optimisation and Predictive Maintenance of Distillation Columns," in Petroleum Refineries and Chemical Plants, Proceedings of the International Nuclear Conference, 2002, Kuala Lumpur, Malaysia.

[2] J. Charlton, Radioisotope Techniques for Problem-Solving in Industrial Process Plants, Leonard Hill, 1986. 
[3] G. Maggio, R. Gillen y J. Graiño, Medidor de interfases para torre de agua pesada, Buenos Aires, CNEA, 1980.

[4] International Atomic Energy Agency, "Radiotracer Technology for Engineering Unit Operation Studies and Unit Processes Optimization”, Technical Report, Krakow, Poland, 1999.

[5] International Atomic Energy Agency, "Radioisotope Applications for Troubleshooting and Optimizing Industrial Processes”, Technical Report, March 2002.

[7] Safety series N³8. IAEA, "Radiation Protection Procedures", Viena, 1973.

[8] S. Glasstone y A. Sesonske, "Ingeniería de Reactores Nucleares”, Barcelona, Editorial Reverté, 1968.

[9] S. Calvo et al., "Guía para Aplicaciones Industriales de Radiotrazadores y Fuentes Selladas de Radiación”, OIEA-ARCAL XLIII, 2000.

[10] S. Calvo, G. Maghella, E. Mamani and J. Maguiña, "Overview of radiotracer experiments for better understanding of wastewater and water treatment plants in Lima (Perú)”, Revista del INCQ. 